\title{
Repeated lumbar punctures within 3 days may affect CSF biomarker levels
}

\author{
Martin Olsson ${ }^{1,2,3,4^{*}}$ (D), Johan Ärlig ${ }^{1,2,3}$, Jan Hedner ${ }^{3,5}$, Kaj Blennow $^{1,2}$ and Henrik Zetterberg $1,2,5,6,7$
}

\begin{abstract}
Lumbar puncture (LP) is a common way of collecting cerebrospinal fluid (CSF) both in the clinic and in research. In this extension of a study on the relationship between sleep deprivation and CSF biomarkers for Alzheimer's disease, we investigated CSF biomarker dynamics in relation to rebound sleep after sleep deprivation. Two LPs were performed within 3 days in 13 healthy volunteers. We noticed an unexpected sharp rise in biomarker concentrations in the second sample and therefore repeated the experiment, but without sleep intervention, in four additional individuals. The findings were similar in these subjects, suggesting an inherent methodological problem with repeated LPS. The result corroborates findings in studies with repeated CSF collection via indwelling lumbar catheters, and needs to be addressed in, for instance, pharmacodynamic studies employing these techniques.
\end{abstract}

Keywords: Lumbar puncture, Cerebrospinal fluid, Alzheimer's disease, Biomarkers, Amyloid B, Tau, GFAp, Neurofilament light, YKL-40

\section{Introduction}

Lumbar puncture (LP) is a routine procedure used in the clinical setting, as well as in research, to collect cerebrospinal fluid (CSF). A wide range of biomarkers can be measured in CSF, making LP a useful procedure in diagnostics of and research on traumatic injuries, infections, autoimmune conditions, bleeding and other CNS pathologies. More specifically, LP has emerged as a key tool in the diagnosis of neurodegenerative disease [1].

We have previously examined the link between partial sleep deprivation and biomarkers for Alzheimer's disease (AD) in both CSF and plasma [2, 3]. In that research setting, with repeat LPs, we also observed a marked increase in several, but not all, brain biomarkers in the sample tapped at the second LP taken 3 days later. This was in consistency with the continuous increase in CSF A $\beta$ levels reported during repeat CSF sampling via an indwelling lumbar catheter during $36 \mathrm{~h}$ [4]. In this paper, we report on these findings and discuss possible mechanisms.

\footnotetext{
${ }^{*}$ Correspondence: martin.olsson@gu.se

${ }^{1}$ Clinical Neurochemistry Laboratory, Sahlgrenska University Hospital,

House V, 43180 Mölndal, Sweden

Full list of author information is available at the end of the article
}

\section{Materials and methods}

In a previously published study [2], 13 healthy adults (Table 1) with normal sleeping habits were exposed to five nights of controlled normal sleep and five nights of restricted sleep ( $<4 \mathrm{~h}$ of sleep). LP was performed in the morning following each period. In an ad-hoc experiment on recovery sleep, another LP was performed $72 \mathrm{~h}$ after the post-sleep deprivation LP. The participants were allowed to sleep freely during this period. Caffeine, nicotine or other central stimulating agents were not allowed. Because of a suspected artefact the data from the ad-hoc data was not published, but instead, a follow-up study was planned.

In the follow-up experiment, four healthy adults (Table 1) with the same inclusion and exclusion criteria as in the original study were subjected to two consecutive LPs in the morning, 3 days apart and without any other intervention. Sleep was monitored by actigraphy (Fig. 1).

\section{CSF sampling and analysis}

CSF samples were collected by LP at the L3/L4 or L4/ L5 interspace with a $22 \mathrm{gx} 90 \mathrm{~mm}$ Sprotte $^{\mathrm{TM}}$ needle, by an experienced neurologist. The needle type was chosen to minimize the risk of post-LP headache [5]. Sampling was 
Table 1 Anthropometric and baseline data

\begin{tabular}{lcc}
\hline Variable & Mean (SD) & \\
\cline { 2 - 3 } & $\begin{array}{l}\text { Recovery sleep } \\
\text { cohort, } \mathbf{N}=\mathbf{1 3}^{\mathbf{a}}\end{array}$ & $\begin{array}{c}\text { Repeated LP } \\
\text { cohort, } \mathbf{~ = 4 ~}\end{array}$ \\
\hline Anthropometric variable & & \\
Age, years & $25(4.0)$ & $25(1.3)$ \\
Weight, kg & $79.3(13.6)$ & $75.8(12.1)$ \\
Height, cm & $184.2(14.0)$ & $181.0(16.1)$ \\
BMl, kg/m² & $23.4(2.4)$ & $23.3(3.3)$ \\
Pulse, bpm & $60(6)$ & $67(9)$ \\
Systolic BP, mmHg & $134(5)$ & $128(9)$ \\
Diastolic BP, mmHg & $81(6)$ & $80(6)$ \\
ESS & $6(3)$ & $2.8(3.3)$ \\
Baseline characteristics & No (\%) & \\
Gender, male & $9(69.2)$ & $0(0.0)$ \\
Nicotine, smoker & $3(23.1)$ & $0(0.0)$ \\
Alcohol, >15 standard units & $1(6.2)$ & \\
\hline
\end{tabular}

$B M I$ body mass index, BP blood pressure, ESS Epworth Sleepiness Scale

a Previously published data [2]

performed between 8 and 9 am 3 days apart. A total of 10-12 mL of CSF was collected in polypropylene tubes, centrifuged at $1300 \mathrm{~g}$ for $10 \mathrm{~min}$, aliquoted and stored in $0.5 \mathrm{~mL}$ aliquots at $-80{ }^{\circ} \mathrm{C}$ pending analysis within $1 \mathrm{~h}$ after sampling.

CSF $A \beta 38, A \beta 40$ and $A \beta 42$ concentrations were measured using MSD Abeta Triplex (Meso Scale Discovery,
Rockville, Maryland). CSF total tau (T-tau) and phosphorylated tau (P-tau) concentrations were measured using INNOTEST sandwich enzyme-linked immunosorbent assays (ELISAs, Fujirebio, Ghent, Belgium). CSF neurofilament light (NF-L) concentration was measured using the NF-Light ELISA (UmanDiagnostics, Umeå, Sweden). CSF YKL-40 (also called chitinase 3-like 1) concentration was measured using the Human Chitinase 3-like 1 Quantikine ELISA Kit (R\&D Systems, Inc. Minneapolis, MN). CSF glial fibrillary acidic protein (GFAP) concentration was measured using an in-house ELISA [6]. All measurements were performed in one round of experiments with one batch of reagents and baseline and follow-up samples side by side on the assay plates by board-certified laboratory technicians who were blinded to clinical data.

\section{Sleep surveillance}

ActiGraph GT3X+ devices were worn on the nondominant wrist, throughout the experiment. Data from the devices was used to validate a normal sleep pattern with at least $8 \mathrm{~h}$ of bedtime. Actigraphy data was reviewed with the ActiLife software and analyzed with the Sadeh algorithm $[7,8]$.

\section{Statistics}

Statistical analysis was done using IBM SPSS version 25.0. Significance was calculated by two-tailed paired sample t-test and alpha was set to 0.05 .

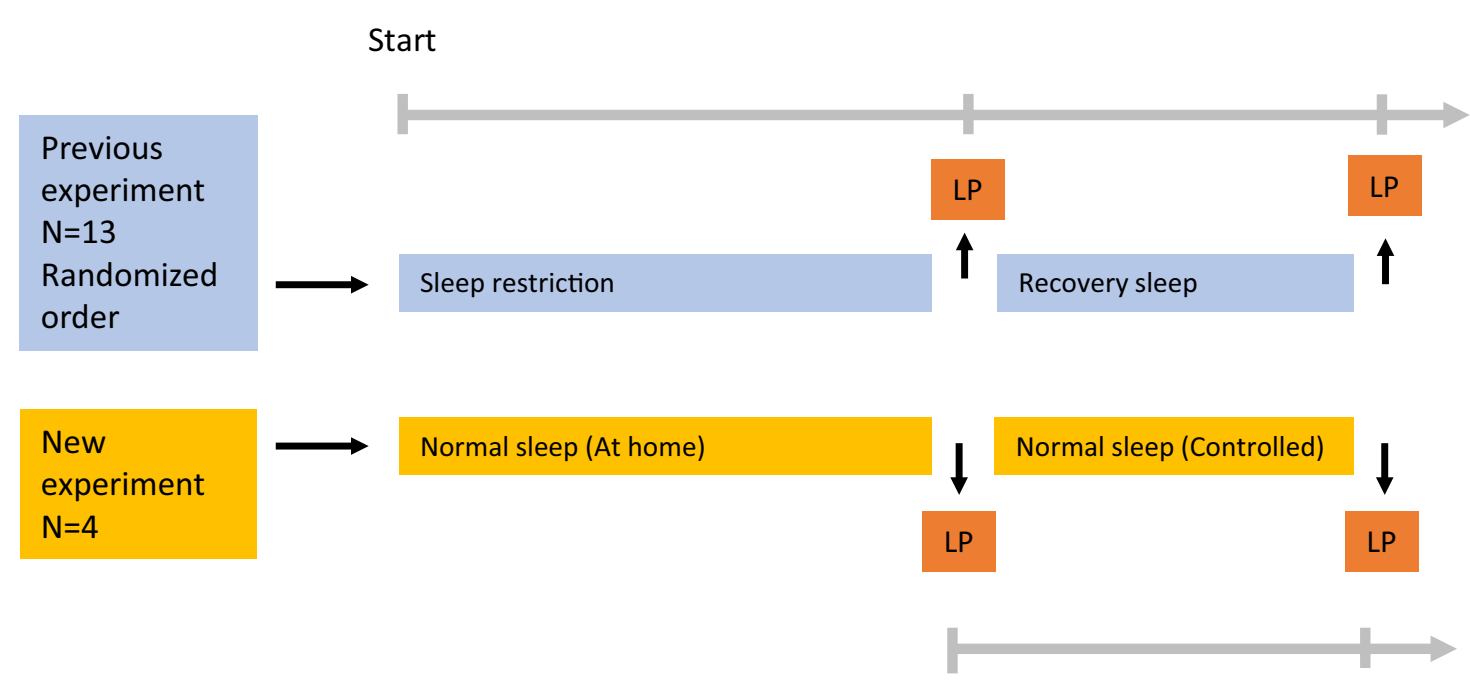

Start

Day 3

Fig. 1 Study flowchart. LP lumbar puncture 


\section{Results}

In conjunction with our earlier experiment, we investigated the effect of recovery sleep on a set of AD biomarkers (Fig. 1). An unexpectedly sharp increase in CSF concentrations of several AD biomarkers after recovery sleep was observed in the sample collected 3 days after the baseline LP (Fig. 2, Table 2). A similar, but less sharp increase in CSF biomarker concentrations was evident in CSF obtained 3 days after a first LP following normal sleep exposure. In contrast, NF-L and GFAP did not change significantly in either the original or the ad hoc protocol.

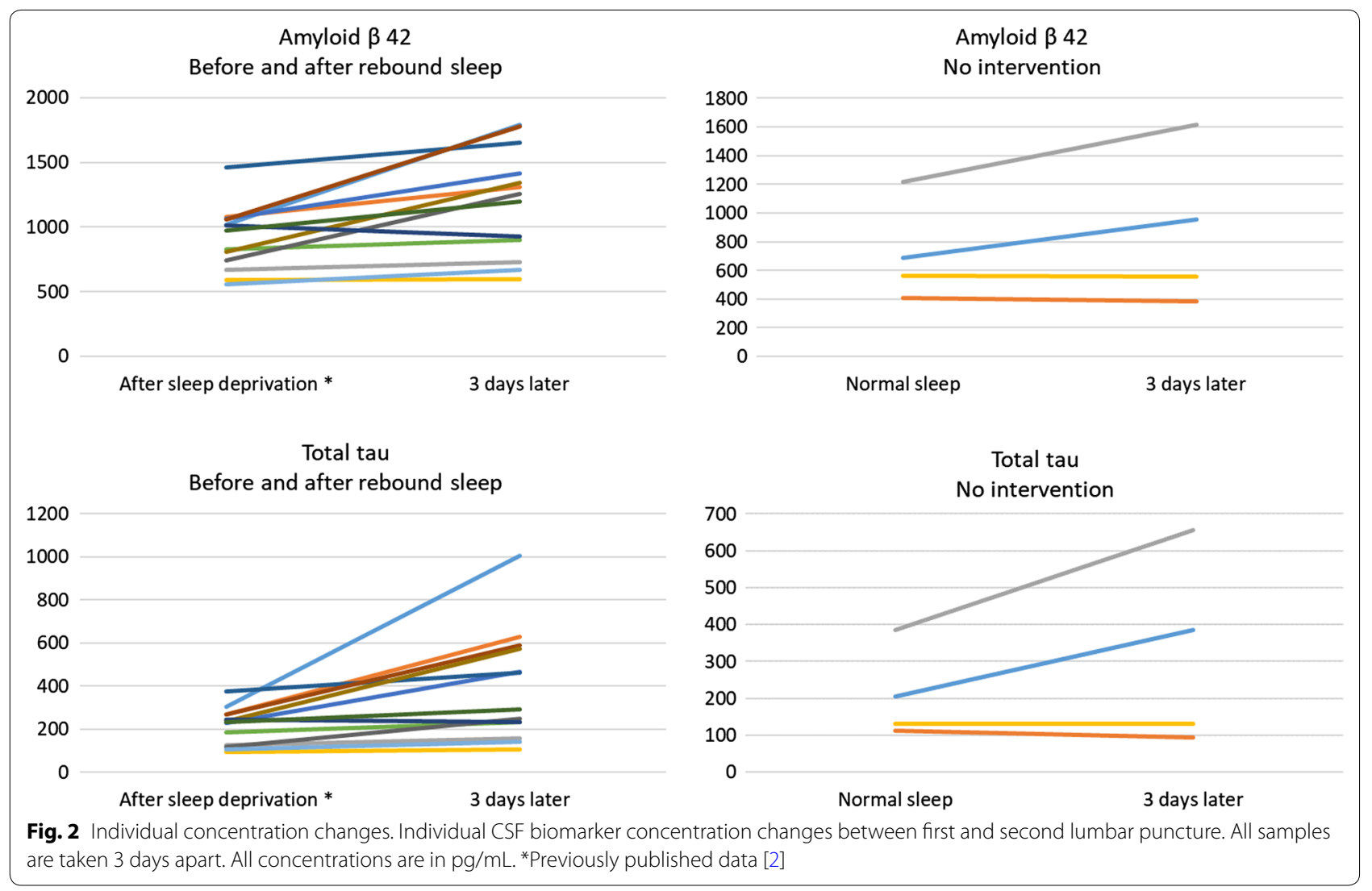

Table 2 CSF biomarker concentrations

\begin{tabular}{|c|c|c|c|c|c|c|c|c|}
\hline & \multicolumn{4}{|c|}{ Recovery sleep } & \multicolumn{4}{|c|}{ No sleep intervention } \\
\hline & $\operatorname{LP} 1 \bar{x}^{a}$ & LP $2 \bar{x}$ & FC & $p$ & LP $1 \bar{x}$ & LP $2 \bar{x}$ & FC & $p$ \\
\hline NFL & 325 & 305 & 0.94 & 0.231 & 198 & 194 & 0.98 & N/A \\
\hline GFAp & 183 & 188 & 1.03 & 0.641 & 236 & 231 & 0.98 & $\mathrm{~N} / \mathrm{A}$ \\
\hline T-tau & 215 & 395 & 1.83 & 0.008 & 208 & 317 & 1.52 & N/A \\
\hline P-tau & 39 & 59 & 1.51 & 0.006 & 31 & 43 & 1.39 & $\mathrm{~N} / \mathrm{A}$ \\
\hline Ab38 & 2640 & 3611 & 1.37 & 0.005 & 2353 & 2930 & 1.25 & N/A \\
\hline Ab40 & 7432 & 9434 & 1.27 & 0.006 & 6249 & 7117 & 1.14 & N/A \\
\hline Ab42 & 913 & 1198 & 1.31 & 0.003 & 719 & 878 & 1.22 & N/A \\
\hline YKL-40 & 56,718 & 72,363 & 1.28 & 0.039 & 56,978 & 71,639 & 1.26 & N/A \\
\hline
\end{tabular}

LP $1 \bar{x}$ and LP $2 \bar{x}$ represent average absolute concentrations in the first and second lumbar puncture, measured in pg/mL. $p$ represents probability value calculated by paired two-tailed t-test

YKL-40 chitinase-3-like protein, NFL neurofilament light, GFAp glial fibrillary acidic protein, $T$ Tau total tau. $P$-tau phosphorylated tau FC fold change of each biomarker

a Previously published data [2] 


\section{Discussion}

The rise in the concentration of several AD biomarkers suggests an inherent methodological problem with repeated LPs within a few days. Studies analyzing repeat CSF samples taken through an indwelling lumbar catheter also report marked increases in $A \beta$ levels, up to $>100 \%$ of baseline levels during the first $12 \mathrm{~h}$, and then fluctuating around double baseline levels for $36 \mathrm{~h} \mathrm{[4].} \mathrm{A} \mathrm{plausible} \mathrm{explanation} \mathrm{is} \mathrm{that} \mathrm{the}$ large volume of CSF tapped (in total $6 \times 36=216 \mathrm{~mL}$ ) may cause a disturbance in the CSF dynamics (flow of CSF from the cerebrum down to the lumbar sac), as suggested by a study showing that a lower CSF sampling frequency (and thus lower CSF volume tapped) reduced such effects on $A \beta$ levels [9]. Alternative explanations include that CSF levels of brain biomarkers are not homogeneously distributed within the CSF space. Among the tested peptides, $A \beta$ (all isoforms) and tau (both total and phosphorylated) have particularly high expression in the cerebrum, as opposed to NFL and GFAp, the expression of which is more evenly distributed throughout the CNS. With high volumes of CSF tapped from the lumbar region, redistribution of CSF originating from regions with close proximity to the cortex to the lumbar sac [9] may occur.

In the present study, we found similar, but less pronounced (only $12 \mathrm{~mL}$ of CSF was tapped at the first LP) increases in CSF levels of brain biomarkers, which may have similar explanations. Contributing explanations may be CSF leakage after the LP into the underpressured epidural space; an explanation supported by the finding that subjects with post-procedure headache (that is known to be caused by CSF leakage) had a more marked increase in CSF concentrations of $A \beta$ than those without this complication [9]. A limitation of the present study is the small number of study objects making significance testing less meaningful. Further, better powered, studies specifically investigating the proposed effect are thus needed.

We would now like to alert the research community of an apparent effect of repeated LPs on CSF biomarker concentrations. It is also worth noting that we used a "non-traumatic" needle type. Using standard needles might have aggravated the biomarker changes even further. These results are important to consider when designing studies with repeated LPs. They may also be of relevance in studies where spinal catheters are placed to examine dynamic changes in CSF composition.

\section{Conclusion}

An LP in itself may have profound effects on CSF protein concentrations if samples are collected by repeated LPs 3 days apart. This confounding influence needs to be taken into account in CSF biomarkers studies with repeat LPs, such as pharmacodynamic Phase I trials.

\section{Abbreviations}

$A \beta$ : amyloid $\beta$; AD: Alzheimer's disease; CSF: cerebrospinal fluid; GFAP: glial fibrillary acidic protein; LP: lumbar puncture; NF-L: neurofilament light.

\section{Acknowledgements}

The authors would like to show regards to the Swedish Research Council, the European Research Council, Swedish State Support for Clinical Research, the Knut and Alice Wallenberg Foundation, the Torsten Söderberg Foundation, Frimurarestiftelsen and the Alzheimer Foundation., for their financial support. Furthermore, we would like to show our appreiciation to Davoud Eskandari, Ding Zou, Ludger Grote, Pontus Wasling, Celia Hök Frohlander, Ann-Chistin Lundquist and all the supporting staff at the Neurochemistry laboratory at Sahlgrenska Mölndal, for their generous contributions in time and energy.

\section{Authors' contributions}

$\mathrm{HZ}$ and $\mathrm{MO}$ had full access to the data in the study and take full responsibility of the accuracy of data analysis and the integrity of the data. Study concept and design: $M O, J \ddot{A}, J H, K B, H Z$. Obtained funding: KB, HZ. Study supervision: HZ. Data acquisition, analysis and/or interpretation: $M O, J \ddot{A}, J H, K B, H Z$. Statistical analysis: MO, HZ. Drafting of the manuscript: MO, HZ. Manuscript revision for critical intellectual content: $M O, J A ̈, J H, K B, H Z$. All authors read and approved the final manuscript.

\section{Funding}

The Swedish Research Council, the European Research Council (Grant No. \#681712), the Knut and Alice Wallenberg Foundation, the Torsten Söderberg Foundation, Frimurarestiftelsen and the Alzheimer Foundation. The funding sources had no role in the design and conduct of the study; collection, management, analysis, or interpretation of the data; and preparation, review or approval of the manuscript; and decision to submit the manuscript for publication. Open access funding provided by University of Gothenburg.

\section{Availability of data and materials}

The datasets generated and/or analysed during the current study are available in the Swedish National Data Service (SND) repository: https://doi. org/10.5878/tpbk-k885, https://doi.org/10.5878/cavp-eh78

\section{Ethics approval and consent to participate}

The study was approved by the Gothenburg Regional Ethical Review Board for Medical Research and was conducted in accordance with the Helsinki declaration. Oral and written informed consent was obtained from all study participants prior to enrolment.

\section{Consent for publication}

Not applicable.

\section{Competing interests}

KB has served as a consultant or at advisory boards for Axon, Biogen, CogRx, Lilly, MagQu, Novartis and Roche Diagnostics. HZ has served at scientific advisory boards for Roche Diagnostics, Wave, Samumed and CogRx, and has given lectures in symposia sponsored by Biogen and Alzecure. $\mathrm{KB}$ and $\mathrm{HZ}$ are cofounders of Brain Biomarker Solutions in Gothenburg AB, a GU Ventures-based platform company at the University of Gothenburg. JH has served as a temporary advisory board member for Merck Sharp and Dohme, Bayer AG, Desitin $\mathrm{GmbH}$, Jazz Pharmaceuticals and Itamar. He is also a co-founder and current board member of CereusScience. Olsson and Ärlig report no disclosures.

\section{Author details}

${ }^{1}$ Clinical Neurochemistry Laboratory, Sahlgrenska University Hospital, House V, 43180 Mölndal, Sweden. ${ }^{2}$ Department of Psychiatry and Neurochemistry, Institute of Neuroscience and Physiology, University of Gothenburg, Mölndal, Sweden. ${ }^{3}$ Center for Sleep and Vigilance Disorders, Institute of Medicine, University of Gothenburg, Gothenburg, Sweden. ${ }^{4}$ Department of Anaesthesiology and Intensive Care Medicine, Sahlgrenska University Hospital/Östra, Gothenburg, Sweden. ${ }^{5}$ Sleep Laboratory, Department of Pulmonary Medicine, Sahlgrenska University Hospital, Gothenburg, Sweden. ${ }^{6}$ Department 
of Neurodegenerative Disease, UCL Institute of Neurology, Queen Square, London, UK. ${ }^{7}$ UK Dementia Research Institute at UCL, London, UK.

Received: 22 October 2019 Accepted: 2 December 2019

Published online: 13 December 2019

\section{References}

1. Molinuevo JL, Ayton S, Batrla R, Bednar MM, Bittner T, Cummings J, et al. Current state of Alzheimer's fluid biomarkers. Acta Neuropathol. 2018;136(6):821-53.

2. Olsson M, Arlig J, Hedner J, Blennow K, Zetterberg H. Sleep deprivation and cerebrospinal fluid biomarkers for Alzheimer's disease. Sleep. 2018;41(5):zsy025.

3. Olsson M, Ärlig J, Hedner J, Blennow K, Zetterberg H. Sleep deprivation and plasma biomarkers for Alzheimer's Disease. Sleep Med. 2019;57:92.

4. Bateman RJ, Wen G, Morris JC, Holtzman DM. Fluctuations of CSF amyloid-beta levels: implications for a diagnostic and therapeutic biomarker. Neurology. 2007;68(9):666-9.
5. Bertolotto A, Malentacchi M, Capobianco M, di Sapio A, Malucchi S, Motuzova Y, et al. The use of the 25 Sprotte needle markedly reduces post-dural puncture headache in routine neurological practice. Cephalalgia. 2015;36:131-8.

6. Rosengren LE, Wikkelso C, Hagberg L. A sensitive ELISA for glial fibrillary acidic protein: application in CSF of adults. J Neurosci Methods. 1994;51(2):197-204

7. Kosmadopoulos A, Sargent C, Darwent D, Zhou X, Roach GD. Alternatives to polysomnography (PSG): a validation of wrist actigraphy and a partialPSG system. Behav Res Methods. 2014;46(4):1032-41.

8. Sadeh A, Sharkey KM, Carskadon MA. Activity-based sleep-wake identification: an empirical test of methodological issues. Sleep. 1994:17(3):201-7.

9. Li J, Llano DA, Ellis T, LeBlond D, Bhathena A, Jhee SS, et al. Effect of human cerebrospinal fluid sampling frequency on amyloid-beta levels. Alzheimers Dement. 2012;8(4):295-303.

\section{Publisher's Note}

Springer Nature remains neutral with regard to jurisdictional claims in published maps and institutional affiliations.
Ready to submit your research? Choose BMC and benefit from:

- fast, convenient online submission

- thorough peer review by experienced researchers in your field

- rapid publication on acceptance

- support for research data, including large and complex data types

- gold Open Access which fosters wider collaboration and increased citations

- maximum visibility for your research: over 100M website views per year

At BMC, research is always in progress.

Learn more biomedcentral.com/submissions 